\title{
INTERNET ENEBLED DATA ACQUISITION AND DEVICE CONTROL
}

\author{
Sandhya D. Foke ${ }^{1}$, R. P. Chaudhari ${ }^{2}$ \\ ${ }^{1}$ Student, Electronics, Government Engineering college Aurangabad, Maharashtra, India \\ ${ }^{2}$ Associate professor, Electronics, Government Engineering college Aurangabad, Maharashtra, India
}

\begin{abstract}
This paper presents very simple and economical way to monitor and control boilers process used in chemical, pharmaceutical companies remotely with the help of internet. Microcontroller ATmega128 is used to which all sensors and devices are interfaced at its I/O pins. A Stand-Alone Ethernet Controller IC which handles most of the network protocol requirements ENC28J60 is interfaced to the microcontroller using SPI interface and acts as a communication media between microcontroller ATmegal 28 and remote user. The TCP/IP stack, application source code, and dynamic web page is stored in microcontroller ATmega128. Dynamic webpage is the key element which eliminates physical presence of user in the field and thus saves the labour time and money.
\end{abstract}

Keywords: ATmega128, ENC28J60, TCP/IP, SPI interface, Dynamic webpage.

$* * *$

\section{INTRODUCTION}

Now a days, world wide web (www) continues to evolve. Enhancement in the embedded technology allows you to use www for controlling and monitoring various devices remotely. Ethernet solves the problem of remote communication with the embedded application.

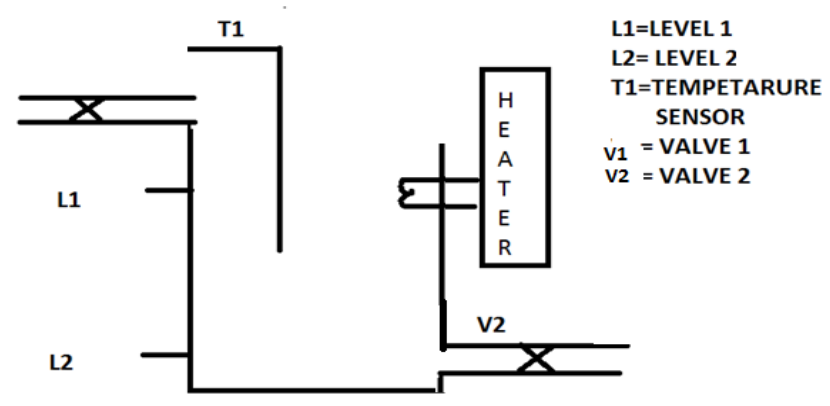

Fig.1: Simple assembly of the project

In this project the heater heats the water which is entered into the tank with the help of input valve. With the help of temperature sensor and level sensor microcontroller controls the switching ON and OFF of the heater and valves. The parameters like temperature and level of the water can be viewed from remote PC using webpage stored in the microcontroller. We can also control valve and heater remotely from webpage. IP address is stored into microcontroller when we enter IP address into the address bar of the internet browser microcontroller will send that webpage with updated data. We can also keep record of past parameters and status of the devices. Dynamic webpage allows you to set threshold points to all parameters.

\section{FUNDAMENTAL COMPONENTS}

- ATmega128

- ENC28J60

- TCP/IP

- SPI interface

- I/O system(Heater, valve, Temperature sensor, Level sensor)

\subsection{MCU ATmega128}

The ATmega128 is 64 pin low power Atmel 8 bit microcontroller. It has advanced RISC architecture with 128 $\mathrm{kb}$ flash program memory which is sufficient to accommodate application source code, dynamic webpage and TCP/IP stack. It has $4 \mathrm{~kb}$ EEPROM and $4 \mathrm{~kb}$ SRAM. It has 133 powerful instructions mostly has single clock cycle execution therefore achieves throughputs approaching 10 MIPS at 16 MHZ[3].

\subsection{ENC28J60}

The ENC28J60 is a stand-alone Ethernet controller with an industry standard Serial Peripheral Interface (SPI $\left.{ }^{\mathrm{TM}}\right)$. It is designed to serve as an Ethernet network interface for any controller equipped with SPI. The ENC28J60 meets all of the IEEE 802.3 specifications. It incorporates a number of packet filtering schemes to limit incoming packets. Communication with the host controller is implemented via two interrupt pins and the SPI, with data rates of up to $10 \mathrm{Mb} / \mathrm{s}$. Two dedicated pins are used for LED link and network activity indication.

The ENC28J60 consists of seven major functional blocks: 1. An SPI interface that serves as a communication channel between the host controller and the ENC28J60.

2. Control registers which are used to control and monitor the ENC28J60. 
3. A dual port RAM buffer for received and transmitted data packets.

4. An arbiter to control the access to the RAM buffer when requests are made from DMA, transmit and receive blocks.

5. The bus interface that interprets data and commands received via the SPI interface.

6. The MAC (Medium Access Control) module that implements IEEE 802.3 compliant MAC logic.

7. The PHY (Physical Layer) module that encodes and decodes the analog data that is present on the twisted pair interface.

The device also contains other support blocks, such as the oscillator, on-chip voltage regulator, level translators to provide $5 \mathrm{~V}$ tolerant I/Os and system control logic [4].

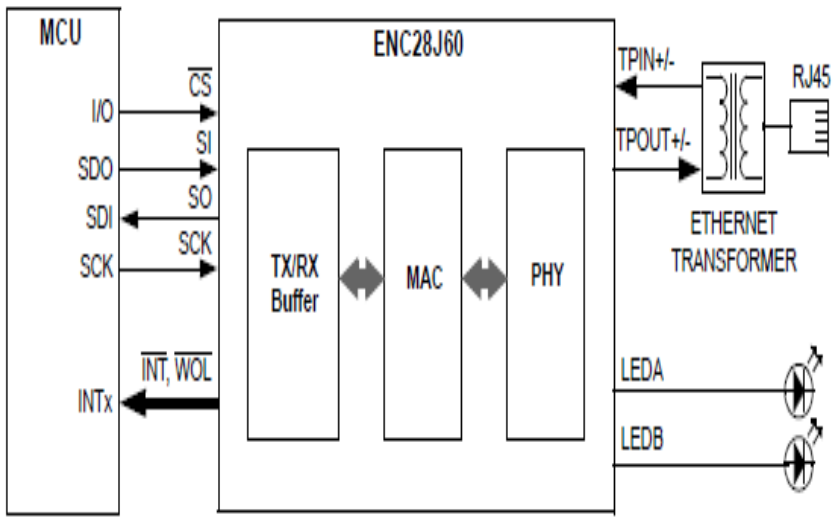

Fig.2: Typical enc28j60 based interface

\subsection{TCP/IP}

The Ethernet standard specifies a way to transfer information between computers in a local network. But Ethernet alone doesn't provide some things that many data transfers require. These include naming the port, or process, that is sending the data, naming the port that will use the data at the destination, handshaking to inform the source whether the destination received the data, flow control to help data get to its destination quickly and reliably, and sequence numbering to ensure that the destination knows the correct order for messages that arrive in multiple segments. The transmission control protocol (TCP) can provide all of these.

TCP uses a system of sequence and acknowledgment numbers that enable the destination to acknowledge receiving specific data bytes. Using sequence numbers, a destination can place received messages in the order they were sent, even if they were received out of order. Sequence numbers also enable a destination to detect duplicate received data. For more efficient transfers of large amounts of data, TCP specifies a way for the source to match the amount of data sent with the ability of the destination to accept new data [1].

\subsection{SPI Interface}

The SPI has a 4-wire synchronous serial interface. Data communication is enabled with a low active Slave Select or Chip Select Signal (SS) or (CS). Data is transmitted with a 3 wire interface consisting of wires for serial data input (MOSI), serial data output (MISO) and serial clock (SCK).

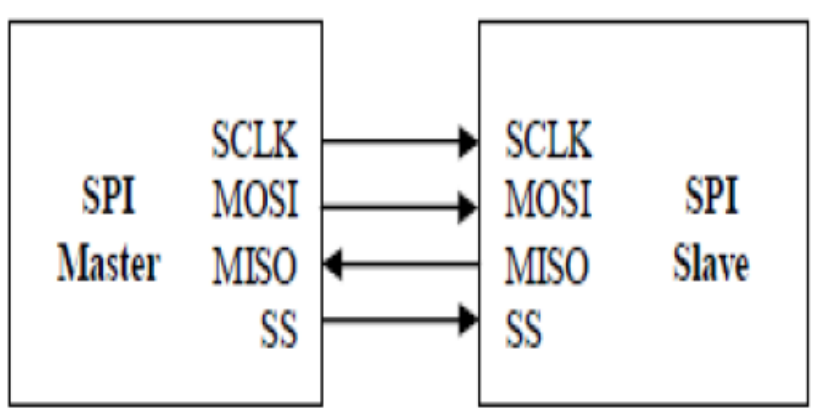

Fig.3: SPI interface

\section{$2.5 \mathrm{I} / \mathrm{O}$ System}

This is simple circuitry to monitor and control different parameters and devices in the field. It is interfaced with the microcontroller using different $\mathrm{I} / \mathrm{O}$ ports. Microcontroller Atmega128 controls the heater and I/O solenoid valves with the help of RTD Temperature sensor and float type level sensor. We can also set the minimum and maximum temperature for the water in the boiler tank.

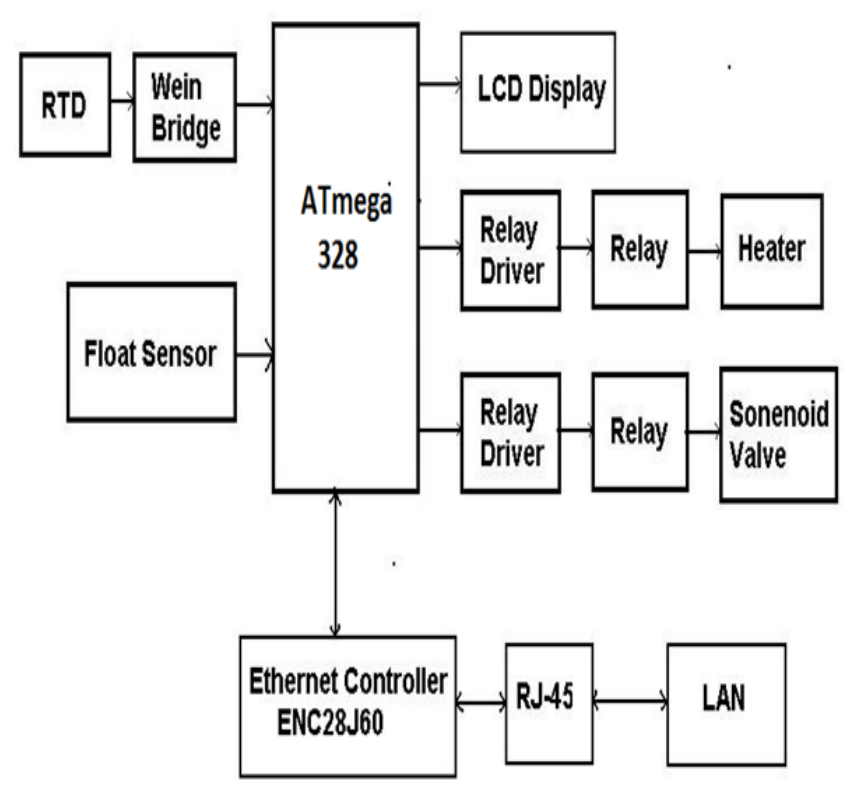

Fig 4: Block diagram of the system 


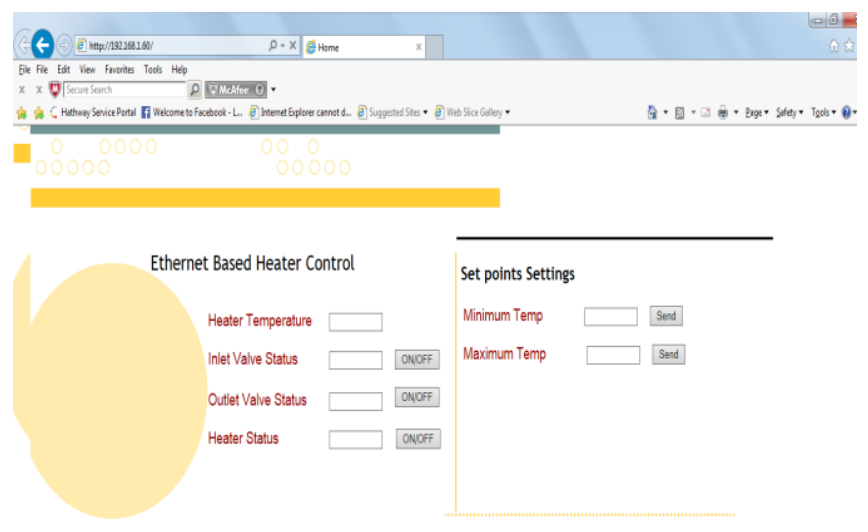

Fig.5: User interface in internet browser

\section{SOFTWARE REQUIREMENT}

The mikroC PRO for AVR is a powerful, feature-rich development tool for AVR microcontrollers. It is designed to provide the programmer with the easiest possible solution to developing applications for embedded systems, without compromising performance or control [7].

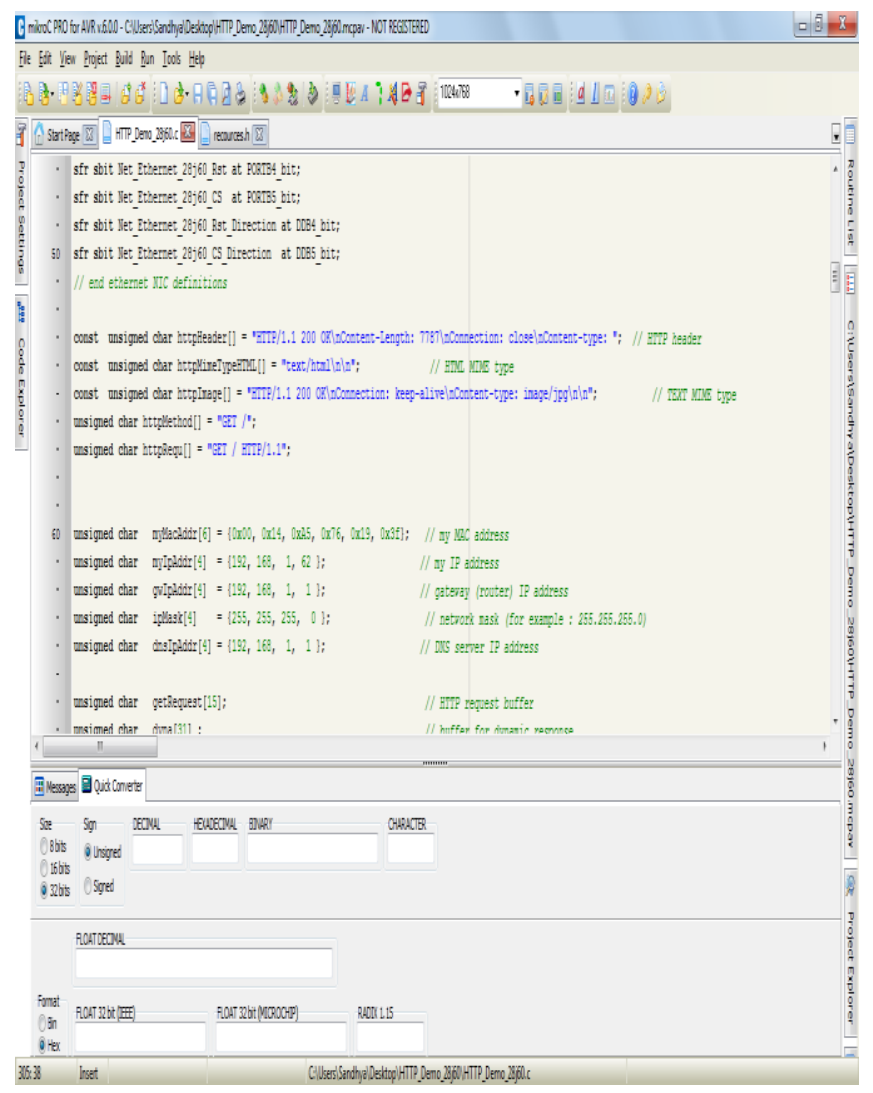

Fig. 6: IDE for avr application

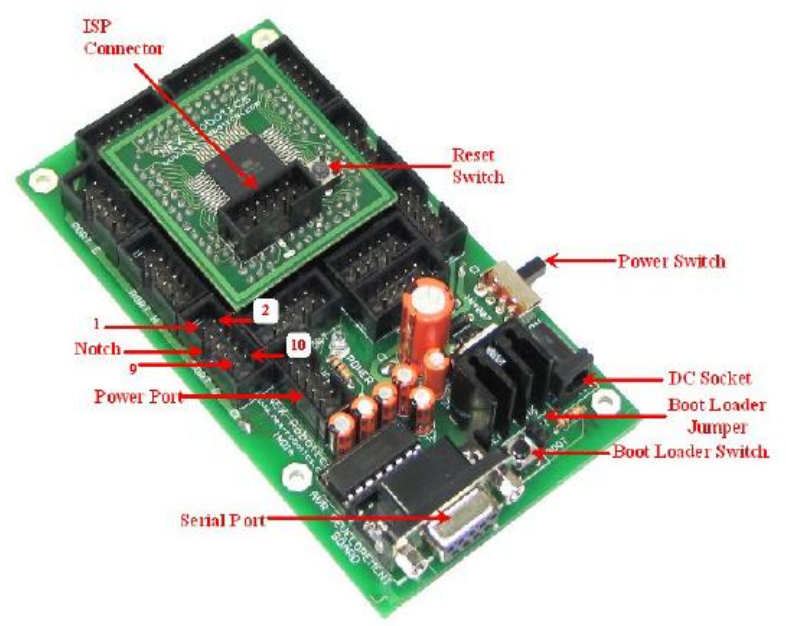

Fig 7: Development board for avr

\section{CONCLUSIONS}

Internet enabled data acquisition and device control system is designed for multiple input and output arrangements for industrial as well as non industrial applications. The system allows you to monitor boiler process through LAN and there is no need to be present physically in the field. It has high speed, low latency, low power consumption. It has different input and output pins for connecting various industrial sensors and switches. The system is small, simple and flexible which is suitable for any industrial application.

\section{REFERENCES}

[1]. Jan Axelson,"Embeded Ethernet and interney complete'2003

[2]. Andrew Tanenbaum,"Computer Networks" 4th edition 2003

[3]. Amel Corporation ," ATmega128p data sheet"2011

[4]. Microchip technology Inc. "ENC28J60 data sheet"2008

[5]. W3schools.com

[6]. www.embeddedmarket.com

[7]. MikroC Pro for AVR, December 2008

[8]. Emily Vander Veer , "Java Scripts for dummies " 4th edition

[9]. Basanta Mahato, Sachin Sharma, Gaurav Chitranshi, "An Embedded Web controllable heater interface for Industry application", 2010 Annual IEEE India Conference (INDICON), 978-1-4244-90745/10/\$26.00 @2010 IEEE

\section{BIOGRAPHIE:}

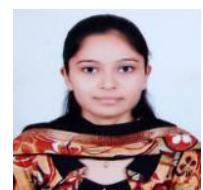

Name: Sandhya D. Foke

Designation: Student

Department: Electronics

Qualification: M.E. Appeared 